\title{
Automated Fuzzy Bidding Strategy Using Agent's Attitude and Market Competition
}

\begin{abstract}
This paper designs a novel fuzzy competition and attitude based bidding strategy (FCA-Bid), in which the final best bid is calculated on the basis of the attitude of the bidders and the competition for the goods in the market. The estimation of attitude is based on the bidding item's attribute assessment, which adapts the fuzzy sets technique to handle uncertainty of the bidding process as well it uses heuristic rules to determine attitude of bidding agents. The bidding strategy also uses and determines competition in the market (based on the two factors i.e. no. of the bidders participating and the total time elapsed for an auction) using Mamdani's Direct Method. Then the final price of the best bid will be determined based on the assessed attitude and the competition in the market using fuzzy reasoning technique.
\end{abstract}

\section{Introduction}

Online auctions have become increasingly important area of research with its popularity, because it provides the traders the flexibility of time and geographical location for trading. Software agent technology is one of the most popular mechanisms used in on-line auctions for buying and selling the goods. Software agent is a software component that can execute autonomously, communicates with other agents or the user and monitors the state of its execution environment effectively [1, $2,3]$. The agents can use different auction mechanisms (e.g. English, Dutch, Vickery etc.) for procurement of goods or reaching agreement between agents. The agent makes decisions on behalf of consumer and endeavors to guarantee the delivery of item according to the buyer's preferences. In these auctions buyers are faced with difficult task of deciding amount to bid in order to get the desired item matching their preferences. The bidding strategies for the software agents can be static or it may be dynamic [4]. The static agents may not be appropriate for the negotiating market situations like extent of competition may vary as traders leave or enter into the market, deadlines and new opportunities may increase the pressure. The dynamic or we can say flexible negotiation capabilities for software agents in the online auctions have become a central concern [5]. Agents need to be able to prepare bids and evaluate offers on behalf of the users they represent with the aim of obtaining the maximum benefit [6] for their users according to the changing market situation.

Much research has already been done by the researchers to formulate different bidding strategies according to the changing market situations $[7,8,9,10$, 11]. Strategies based on flexible negotiation agents perform better as compared to the 
strategies based on fixed negotiation agents [5,12]. Faratin et al in [13] developed strategies based on time, attitude, resources, but many more factors such as competition, trading alternatives are not considered.

In this paper we focus on the design of a novel bidding strategy based on the above mentioned factors to be used by the software agent in online auction. A fuzzy competition and attitude based bidding strategy(FCA-Bid) is designed, in which the final best bid is calculated on the basis of the attitude of the bidders as well as the competition for the goods in the market.

\section{Fuzzy Competition and Attitude Based Bidding Strategy (FCA-Bid)}

The agent's decision making about bidding involves various internal and external environmental factors. The internal factors include good or item's attributes, attitude of the agents on the assessment of attributes, and current available number of the goods and the external environmental factors may include like competition for the goods in the market, nature of the market supply (demand), other opportunities available in the market and many more.

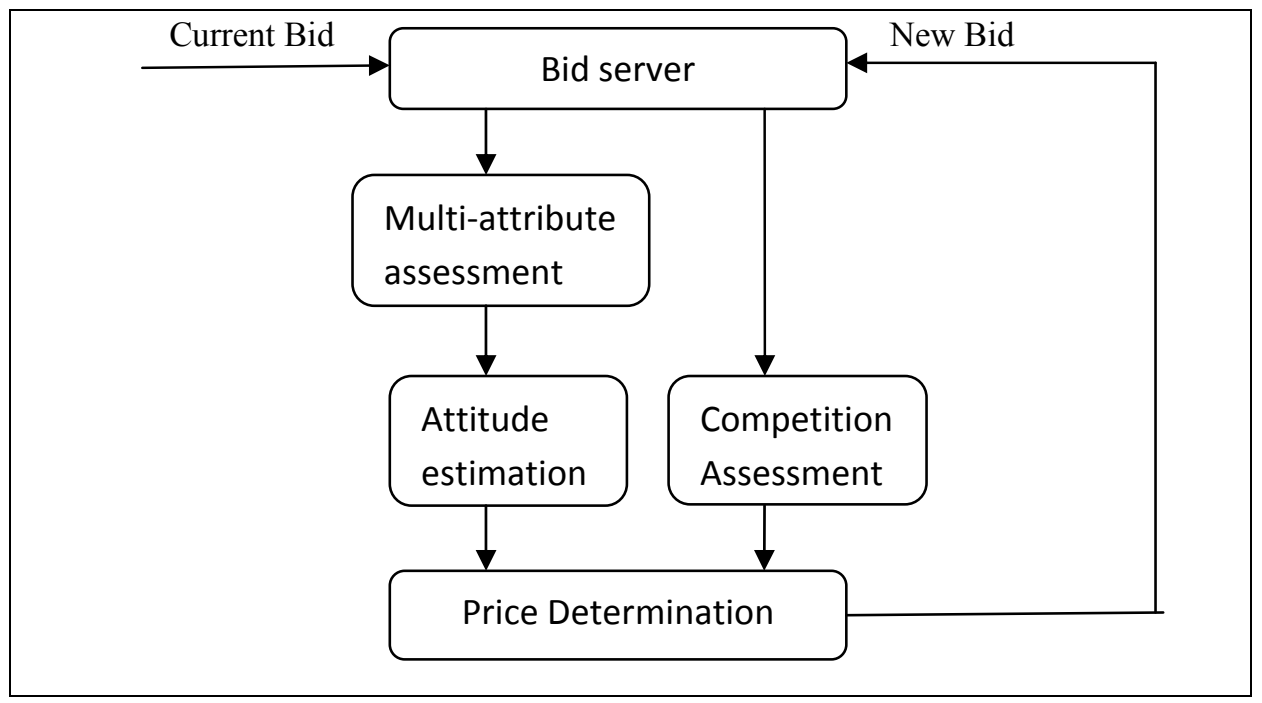

Fig. 1. A Fuzzy Bidding Strategy (FCA-Bid) Model

In fuzzy competition and attitude based bidding strategy (FCA-Bid) (Fig. 1), the factors which are focused are attitude of the agents with respect to the goods' attributes and competition for the goods in the market. For estimation of the price for a bid for winning an auction, the agent must have a balanced behavior between these factors i.e. the attitude (eagerness) to win the auction based on the attributes of the goods and finding the competition for the goods in the market. The attitude towards bidding the quality goods is more as compared to the less quality goods. The bidding 
price also affects the attitude of the agents. The higher bid price dampens the attitude of the agents towards the goods. Also the increasing competition for the goods in the market increases the attitude for that good. The competition in turn depends on the number of bidders and the time elapsed for the auction. As the number of bidders increases, the competition among them also increases, resulting in a higher price. In the beginning of the auction the competition is less and it increases as time elapses and it is at the peak when time approaches approximately in the middle of the auction period. At the end of the auction period the competition among the bidders decreases. The steps of the design of fuzzy competition and attitude based bidding strategy (FCA-Bid) are as follows:

- first, each attribute is evaluated and then the assessment of all these attributes will be aggregated

- then attitude of the agent will be found based on these assessments,

- next the level of competition as the function of no. of bidders and time elapsed for the auction will be found

- Finally the best bid is calculated on the basis of the above attitude of the agents and the competition for the goods in the market.

In this paper we have used fuzzy set methods to deal with the uncertainty, which exists during the determination of overall assessment of the goods for their attributes, the attitude of the agent based on the assessment of goods and the level of competition in the market. First of all, this paper uses a satisfactory degree measure as the common universe of assessment, i.e., an assessment is treated as a fuzzy set on the satisfactory degree. Secondly, an attitude is expressed as a fuzzy set on the set of assessments, i.e., the assessment set is the universe of attitude (eagerness). Thirdly, competition is expressed as a fuzzy set on the fuzzy sets of the no. of bidders and the time elapsed of the auction.

\subsection{Attribute Evaluation}

The attribute evaluation is done in two parts [13]. First the expression for the assessment of the attributes is found then these assessments will be aggregated to find the overall assessment of the attributes of the goods. Let $C=\left\{C_{0}, C_{1}, \ldots, C_{K}\right\}$ be the set of $K+1$ attributes and $W=\left\{w_{0}, w_{1}, \ldots, w_{k}\right\}$ is the set of weights for attributes in $C$.

Attribute Assessment The assessment of the attributes is expressed in terms of a fuzzy set. Let $A=\left\{a_{1}, a_{2}, \ldots \ldots \ldots, a_{n}\right\}$ be a set of assessment terms on the universe i.e. the satisfactory degree[0,1]. This is the satisfactory degree of the agent to a particular attribute. All the fuzzy sets have same universe which is convenient for the aggregation of various assessments. Let $g_{k}(k=0,1, \ldots, K)$ is the satisfactory degree measure for attribute $c_{k}$. Then an agent's opinion on the goods in terms of attribute $c_{k}$ is denoted by $g_{k}(u)$ where $u\left(\in U_{k}\right)$ is the real attribute value of attribute $c_{k}$ and $U_{k}$ is the real universe for attribute $c_{k}$. For instance, departing time is an attribute for a flight ticket. The possible departing time in a day is from 0:00 to 23:59. For any time slot $u$, a client may present a satisfactory degree such as departing at 7:30 is with satisfactory 
degree 0.9 and departing at $3: 00$ is with 0.3 . In the following, $A=\left\{a 1, \ldots, a_{n}\right\}$ be the set of used assessment terms which are fuzzy sets on satisfactory degree $[0,1]$. Then a numeric satisfactory degree is transformed to a linguistic term. In the above example [14] $a 7$ is with the biggest the membership degree for 0.9 , the assessment for departing at 7:30 is $a_{6}$ by the maximum membership degree principle. Similarly, the assessment for 0.3 is $a_{2}$.

Aggregation of Assessments All the goods have a number of different attribute. So to find the overall estimation on the good, the assessment of these all attributes will be aggregated together. Take booking a flight ticket for example, an assessment is made on a ticket usually based on the airlines, flight departure and arrival time, flight type, aircraft types, seat positions, as well as price. The change of an attribute's value may leads to the alternation of an assessment. Instinct natures of different attributes increase the difficulty and uncertainty for obtaining an overall assessment. Notice that an agent's preference on an individual attribute can be expressed through the agent's satisfactory degree on that attribute. This paper uses a satisfactory degree measure as the common universe of assessment. Based on assessment on each individual attribute, an overall assessment can be obtained as follows. Suppose the individual assessments of all attributes are $v_{0}, v_{1}, \ldots, v_{K}$ and the weights of them are $w_{0}, w_{1}, \ldots$, $w_{k}$ respectively. Then an overall assessment is obtained by taking the difference between ã and $a_{i} \in \mathrm{A},[14]$ where $\tilde{\mathrm{a}}$ is a fuzzy set on $[0,1]$ as follows

$$
d\left(\tilde{\mathrm{a}}, a_{i}\right)=\int\left|\tilde{\mathrm{a}}-a_{i}\right| \mathrm{d} \lambda .
$$

Finally, we select the nearest term(s) $a$ to ã as the overall assessment.

\subsection{Attitude Estimation}

Attitude is a learned predisposition to respond in a consistently favorable or unfavorable manner with respect to a given object [13][14]. In other words, the attitude is a preparation in advance of the actual response, constitutes an important determinant of the ensuing behavior. In AI, the fundamental notions to generate the desirable behaviors of the agents often include goals, beliefs, intentions, and commitments. The exhibited behavior is based on a number of factors which depends on the nature of the dynamic world. Once an agent chose to adopt an attitude, it strives to maintain this attitude, until it reaches a situation where the agent may choose to drop its current attitude towards the object and adopt a new attitude towards the same object. Thus, an agent's attitude towards an object refers its persistent degree of commitment towards achieving one or several goals associated with the object, which give rise to an overall favorable or unfavorable behavior with regard to that object. In online auctions the attitude of an agent towards the goods is the eagerness that measures agent's interest in negotiating and coming to a deal [14]. The level of interest may be categorized as: must deal, desirable, nice to have, optional, unessential, and absolutely unessential [15]. Attitude is related to the overall assessment on the given goods. It is expected to change as per the changes in internal and external environmental conditions. Like the attitude for the goods having better 
assessment have more positive attitude of bidding those goods and also the attitude towards more competitive goods is stronger. In this paper we will estimate the attitude on the bases of the assessment on the goods and will consider competition as an independent factor in calculating the final bid. After conducting new assessment on the goods according to current price $p_{c}$, estimation of agent's attitude is implemented. In order to do so, the relationship between attitude and assessments is required. As said earlier, the better the assessment on the given goods is, the stronger the attitude of bidding for those goods will be.

Suppose $E=\{e 1, \ldots, e m\}$ is the set of attitude expressions, $A=\left\{a 1, \ldots, a_{n}\right\}$ is the set of assessments, and $T=\{t 1, \ldots, t L\}$ is the agent's transaction records such that $t_{i}=1$ if the client won the transaction $t i$, otherwise $t_{i}=0$. Because in each transaction, the agent's assessment and attitude occur simultaneously, a set of formal rule, denoted by $R$, thus can be extracted from $T$ such that any $r \in R$ is of form

$$
\mathrm{r}:\left(\mathrm{ai}_{\mathrm{i}} \Rightarrow \mathrm{ej}_{\mathrm{j}}, \mathrm{\alpha}_{\mathrm{ij}}\right)
$$

where ai $\in A, e j \in E$, and $\alpha_{i j}$ is the reliability degree obtained by

$$
a_{i j}=\frac{\mid\{t \in T \mid a i, e j \text { occur in } t \text { and } t=1\} \mid}{\mid\{t \in T \mid \text { ai occurs in } t \text { and } t=1\} \mid}
$$

Such rule depicts the approximate degree of agent's attitude e $\mathrm{j}$ to which the agent can win the bid under the assumption that the overall assessment is ai [13]. Furthermore, these rules can be treated as a set of fuzzy sets on A such that the membership degree in a fuzzy set $\mathrm{f}_{\mathrm{j}}$ corresponding to eagerness e $\mathrm{j}$ is $\boldsymbol{\alpha}_{\mathrm{ij}}$. Obviously, $\mathrm{f}_{\mathrm{j}}$ is an integration of rules $\left(a_{i} \Rightarrow e_{j}, \alpha_{i j}\right)(i=1, \ldots, n)$, which is able to be treated as an alias of ej . Hence, the fuzzy set $\mathrm{f}_{\mathrm{j}}$ is also called attitude in the following without other specification. Based on the rules in $\mathrm{R}$, an agent can estimate the possible attitude[13] of the agent when it learns the current overall assessment. Set of fuzzy sets is obtained through the following way: suppose the overall assessment is ac, then the attitude at the moment is determined by the maximum membership degree principle

$$
e_{c} \in E(a c)=\left\{e_{j} \in E \mid f_{j}(a c) \geq f_{i}(a c) \text { if } i \neq j\right\}
$$

Notice that such determined ec may not necessarily be unique. In the following, we call $\mathrm{E}(\mathrm{ac})$ the candidate attitude set under ac.

\subsection{Competition Assessment}

The level of competition in an auction may be captured by the number of bidders and the time elapsed. Competition among bidders plays an integral role in price formation [17]. As the number of bidders increases, the competition among them also increases, (Fig. 2) resulting in a higher price. Bapna, Jank and Shmueli [18] found the number of bidders to be positively associated with the current price of the item. Furthermore, it is observed that, typically, the middle of the auction experiences a smaller amount of bidder participation as compared to the 
early and later stages of the auction. Bidders generally utilize this time to scrutinize the auctioned item or just simply wait to see how other bidders behave. Therefore, it would be interesting to see how this competition characteristic affects the on-line auction's price formation. We anticipate that the number of bidders has a significant positive relationship with price levels. In the beginning of the auction the competition is less and it increases as time elapses and it is at the peak when time approaches approximately in the middle of the auction period. At the end of the auction period the competition among the bidders decreases (Fig. 3). Here we will describe the competition factor in terms of no. of bidders (b) and the total time elapsed ( $\mathrm{t}$ ) for the auction of items. We will consider the competition as a set fuzzy set of values $\mathrm{c}_{1}, \mathrm{c}_{2}, \ldots \ldots \mathrm{c}_{\mathrm{n}}$, no. of bidders $\mathrm{B}$ as a fuzzy set of values $\mathrm{y}_{1}, \mathrm{y}_{2} \ldots \ldots \mathrm{y}_{\mathrm{n}}$. And the time elapsed as another fuzzy set $T$ of values $x_{1}, x_{2}, \ldots . . x_{n}$.

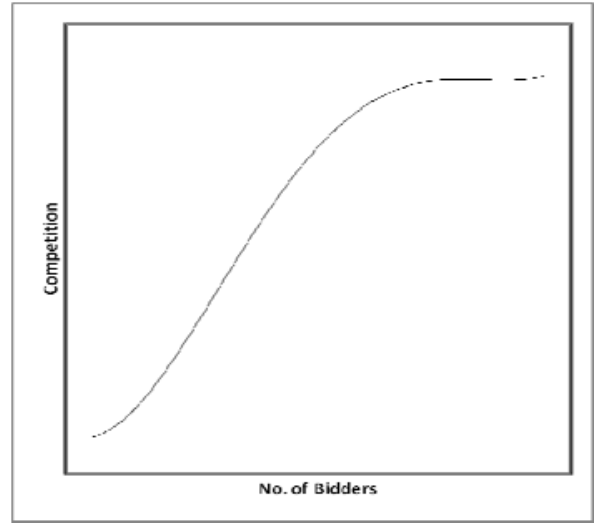

Fig.2. Competition versus Number of Bidders

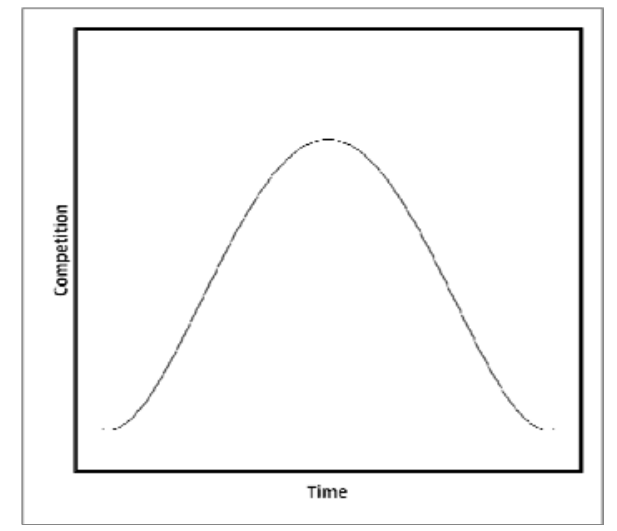

Fig. 3. Competition versus Time Elapsed

According to Mamdani's Direct Method[19] we can find adaptability n no. of rules $w 1, w 2, \ldots \ldots . w n$ as follows

$$
\begin{aligned}
& \mathrm{w} 1=\mu \mathrm{x} 1(\mathrm{~T}) \vee \mu \mathrm{y} 1(\mathrm{~B}) \\
& \mathrm{w} 2=\mu \times 2(\mathrm{~T}) \vee \mu \mathrm{y} 2(\mathrm{~B}) \\
& \mathrm{wn}=\mu \mathrm{xn}(\mathrm{T}) \vee \mu \mathrm{yn}(\mathrm{B}) \\
& \mu \mathrm{c}^{\prime} 1(\mathrm{C})=\mathrm{w} 1 \vee \quad \mu \mathrm{c} 1 \\
& \mu \mathrm{c}^{\prime} 2(\mathrm{C})=\mathrm{w} 2 \vee \quad \mu \mathrm{c} 2 \\
& \mu \mathrm{c} \text { 'n }(\mathrm{C})=\mathrm{wn} \vee \quad \mu \mathrm{cn}
\end{aligned}
$$

Then the conclusion of each rule can be found as follows

These conclusions can be aggregated to find the final conclusion

$$
\mu \mathrm{c}(\mathrm{C})=\mu \mathrm{c}^{\prime} 1(\mathrm{C}) \wedge \mu \mathrm{c}^{\prime} 2(\mathrm{C}) \wedge \ldots \ldots \ldots . . .^{\wedge} \mu \mathrm{c}^{\prime} \mathrm{n}(\mathrm{C})
$$

To find the definite value for the conclusion, here center of gravity of the fuzzy set has been applied as follows 


$$
C=\frac{\int \mu z(C) C d C}{\int \mu z(C) d C}
$$

\subsection{Agent Price Determination}

Price of the goods depends on the attitude towards that good and the competition in the market for that good. If the attitude for the goods is positive and also competition for that product in the market is high then the price of the item is high. If the attitude is negative and competition is also low then the price for that item is obviously be low. If the attitude is positive and the competition is low then the price is going to be medium and so on.

We can calculate the price of the good based on the assessed attitude and the competition determined which is based on the no. of bidders and time elapsed for the auction by applying Mamdani's Method for fuzzy relations and compositional rule of inference[18]. Here we will describe the price of goods in terms of attitude of agent towards the good and competition in the market for that good. We will consider bid displacement factor $\Delta \mathrm{P}$ as a fuzzy set of values $\mathrm{p} 1, \mathrm{p} 2, \ldots \ldots \ldots \mathrm{pn}$, attitudes $\mathrm{E}$ as a fuzzy set of values e1,e2.......en and competition $\mathrm{C}$ as a fuzzy set of values $\mathrm{c} 1, \mathrm{c} 2, \ldots \ldots \ldots \mathrm{cn}$. According to Mamdani's Method for fuzzy relations and compositional rule of inference the rule ei and $\mathrm{cj} \rightarrow \mathrm{pk}$ can described by

$$
\mu \mathrm{R}(\mathrm{E}, \mathrm{C}, \Delta \mathrm{P})=\mu \mathrm{ei}(\mathrm{E})^{\wedge} \mu \mathrm{cj}(\mathrm{C})^{\wedge} \mu \mathrm{pk}(\Delta \mathrm{P})
$$

For $\mathrm{n}$ no. of rules, the compiled fuzzy relation $\mathrm{R}$ is given as

$$
\mathrm{R}=\mathrm{R} 1 \mathrm{UR} 2 \mathrm{U} \ldots \ldots \ldots \ldots \ldots . . . \mathrm{URn}
$$

For the input of fuzzy set E' on $\mathrm{E}$ and fuzzy set $\mathrm{C}$ ' on $\mathrm{C}$, the output fuzzy set $\Delta \mathrm{P}^{\prime}$ on $\Delta \mathrm{P}$ can be obtained as follows

$$
\Delta \mathrm{P}^{\prime}=\left(\mathrm{E}^{\prime} \text { and } \mathrm{C}^{\prime}\right) \mathrm{o} \mathrm{R}=\mathrm{E}^{\prime} \mathrm{o}\left(\mathrm{C}^{\prime} \mathrm{oR}\right)=\mathrm{C}^{\prime} \mathrm{o}\left(\mathrm{E}^{\prime} \mathrm{oR}\right)
$$

and then the final price for the bid will be

Final bid $=$ Current bid $+\Delta \mathrm{P}^{\prime}$

\section{Experimental Evaluations}

In this section, an experiment implements the fuzzy bidding strategy in a scenario in which an agent intends to book flight tickets. Six factors (as shown in Table 1) are 
concerned in this situation, i.e. ticket price (c0), depart time (c1), arrival time (c2), number of stops (c3), seat positions (c4), and travel season (c5). The flight ticket bid for is a return ticket to destination $D$ with the following properties:

- price: $\$ 800-\$ 2000$;

- depart time: 18:00 PM, Wednesday;

- return arrival time: 10:00 AM, Friday;

- number of stops: 1 ;

- seat position: window;

- travel season: April (off-peak season).

Suppose the identified perspective of an agent is summarized as below:

Table 1. Concerned Attributes of a Flight Ticket.

\begin{tabular}{|c|c|c|}
\hline Attributes & Symb. & Values range \\
\hline price & $c 0$ & $\$[800-2000]$ \\
\hline depart time & $c 1$ & Sun. 0:00-Sat. 24:00 \\
\hline arrival time & $c 2$ & Sun. 0:00-Sat. 24:00 \\
\hline stops & $c 3$ & $0,1,2,3$ \\
\hline seat position & $c 4$ & window, aisle, middle \\
\hline flight season & $c 5$ & Jan. 01 - Dec. 31 \\
\hline
\end{tabular}

- The agent prefers to a cheaper ticket and agrees to that the cheaper the better.

- The agent prefers to travel at the weekend rather than at working day.

- The agent prefers to no stop travel.

- The agent prefers to aisle seat then window seat.

- The agent prefers to travel during off-peak season rather than peak season.

- The agent thinks the flight price is the most important factor, secondly the travel season, and other factors are of same importance.

Based on the agent's perspective, the agent evaluates the attitudes using seven terms , very bad (a1), bad (a2), slightly bad (a3), acceptable (a4), fairly good (a5), good (a6), and very good $(a 7)$.The seven terms are expressed by fuzzy sets on the satisfactory degree $[0,1]$ as below

$$
\mathrm{f}_{\mathrm{ai}}=\mathrm{e}^{-162(\mathrm{x}-(\mathrm{i}-1) 1 / 6)^{\wedge} 2}, \quad i=1, \ldots, 7
$$

The assessment on each individual factor is

\begin{tabular}{|l|l|}
\hline Attribute & Assessment \\
\hline c0 & (no assessment) \\
\hline c1 & good $(a 6)$ \\
\hline c2 & fairly good $(a 5)$ \\
\hline c3 & slightly bad $(a 3)$ \\
\hline c4 & acceptable $(a 4)$ \\
\hline c5 & good $(a 6)$ \\
\hline
\end{tabular}


Now, a fuzzy set a $(u)$ is obtained, the most nearest assessment to ã is a6. So the new overall assessment for the ticket is a6 [13].Then the agent needs to estimate the agent's attitude according to this assessment. Suppose the agent uses five terms to distinguish the attitude, i.e., none (e1), slightly (e2), medium(e3), strong (e4), and very strong (e5). In order to estimate the agent's attitude, a set of rules are extracted from a historical auction records, which are illustrated in Table2 and Fig. 4.

Table 2. Rule set for attitude estimation.

\begin{tabular}{|l|c|c|c|c|c|}
\hline & \multicolumn{4}{|l|}{ Attitude } & \multicolumn{3}{l|}{} \\
\hline Ass. & e1 & e2 & e3 & e4 & e5 \\
\hline a1 & 0.17 & 0.23 & 0.2 & 0.27 & 0.13 \\
\hline a2 & 0.1 & 0.28 & 0.22 & 0.26 & 0.13 \\
\hline a3 & 0.1 & 0.26 & 0.18 & 0.32 & 0.13 \\
\hline a4 & 0.17 & 0.26 & 0.23 & 0.23 & 0.12 \\
\hline a5 & 0.12 & 0.25 & 0.27 & 0.21 & 0.16 \\
\hline a6 & 0.12 & 0.26 & 0.26 & 0.23 & 0.13 \\
\hline a7 & 0.12 & 0.24 & 0.31 & 0.24 & 0.1 \\
\hline
\end{tabular}

By Fig. 4, the agent's attitudes at this moment are $e_{2}$ and $e_{3}$ because they have the highest reliability. Because $e 3$ is stronger than $e 2$, the agent first searches possible bids under the attitude $e 3$.

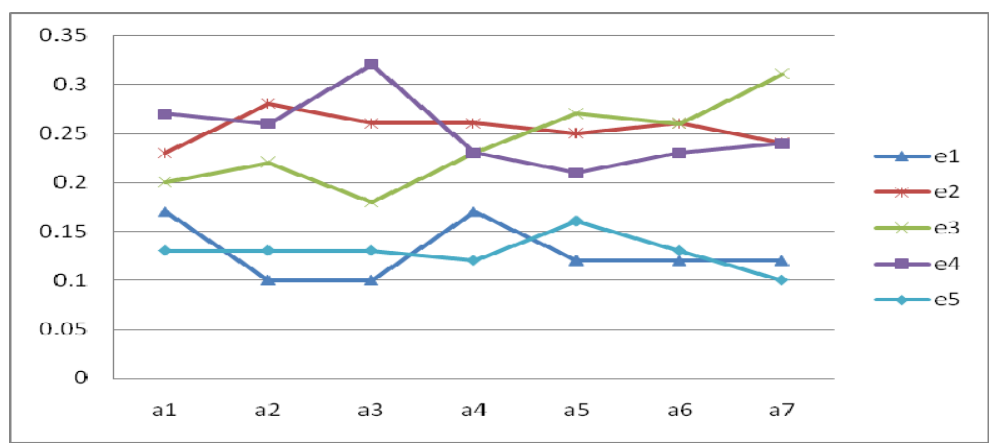

Fig. 4. : Illustration for Rule Set

For finding the price of the good, we will apply fuzzy logic by considering two factors attitude and competition as described in the section 2.4. Let us consider the following set of rules for the logic using various fuzzy sets. 
Rule 1: IF attitude of agent for buying the goods is E1

AND competition in the market for that product is $\mathrm{C} 1$

THEN price for that item will be displaced by $\mathrm{P} 1$

Rule 2: IF attitude of agent for buying the goods is E1

AND competition in the market for that product is $\mathrm{C} 2$

THEN price for that item will be displaced by $\mathrm{P} 2$

Rule 3: IF attitude of agent for buying the goods is E2

AND competition in the market for that product is $\mathrm{C} 1$

THEN price for that item will be displaced by $\mathrm{P} 2$

Rule 4: IF attitude of agent for buying the goods is E2

AND competition in the market for that product is $\mathrm{C} 2$

THEN price for that item will be displaced by $\mathrm{P} 3$

These fuzzy sets represents the linguistic variables as follows: attitudes low as E1 and high as E2, Competition less as $\mathrm{C} 1$ and more as C2 and Negative displacement as P1, no displacement as $\mathrm{P} 2$ and positive displacement as $\mathrm{P} 3$. We assume that the set of attitudes for buying any item as $\mathrm{E}=\{\mathrm{e} 1, \mathrm{e} 2, \mathrm{e} 3\}=\{0,0.5,1\}$ and set of competition for the good in the market as $\mathrm{C}=\{\mathrm{c} 1, \mathrm{c} 2, \mathrm{c} 3\}=\{0,0.5,1\}$. Also, the bid displacement as $\Delta \mathrm{P}=\{\mathrm{p} 1, \mathrm{p} 2, \mathrm{p} 3\}=\{-100,0,+100\}$. The fuzzy sets used in the preceding four rules can be quantized as shown in the Fig. 5:
$\mathrm{E} 1=[1.0,0.5,0]$
$\mathrm{C} 1=[1.0,0.5,0]$
$\mathrm{P} 1=[1.0,0,0]$
$\mathrm{E} 2=[0,0.5,1.0] \quad \mathrm{C} 2=[0,0.5,1.0] \quad \mathrm{P} 2=[0,1.0,0]$
$\mathrm{P} 3=[0,0,1.0]$
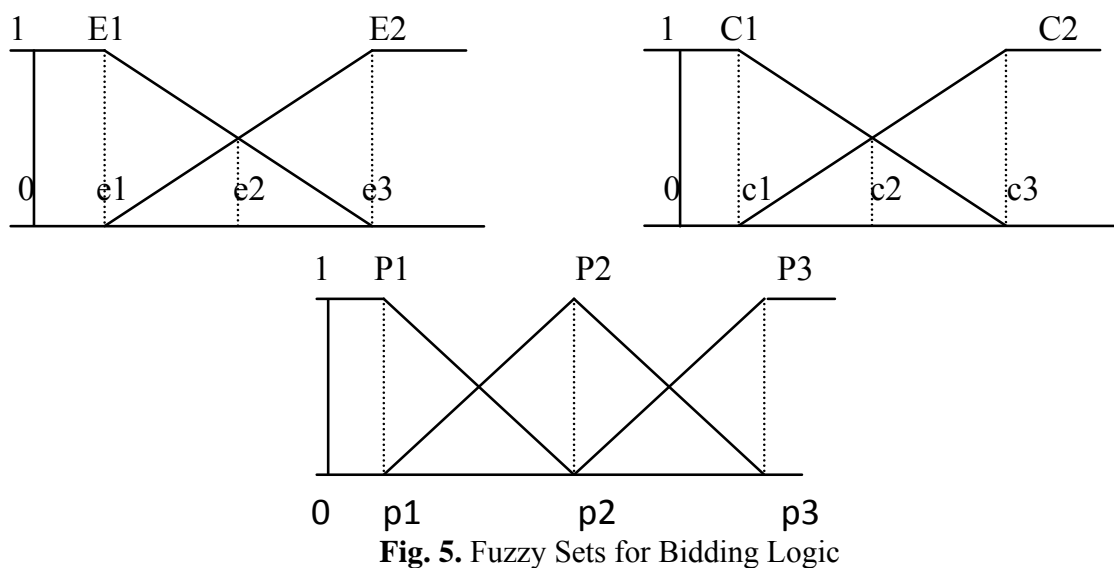

Note that the number of elements in E, C and P are three and the fuzzy sets are also quantized into three elements. Now let us construct fuzzy relations by Mamdani's Method for fuzzy relations

$$
\underset{\mathrm{i}, \mathrm{j}, \mathrm{k}=1,2,3}{\mu \mathrm{R}(\mathrm{E}, \mathrm{C}, \Delta \mathrm{P})=\mu \mathrm{ei}(\mathrm{E})^{\wedge} \mu \mathrm{cj}(\mathrm{C})^{\wedge} \mu \operatorname{pk}(\Delta \mathrm{P})}
$$


By the preceding conversion formula we get the fuzzy relation $\mathrm{R} 1$ from the first rule:

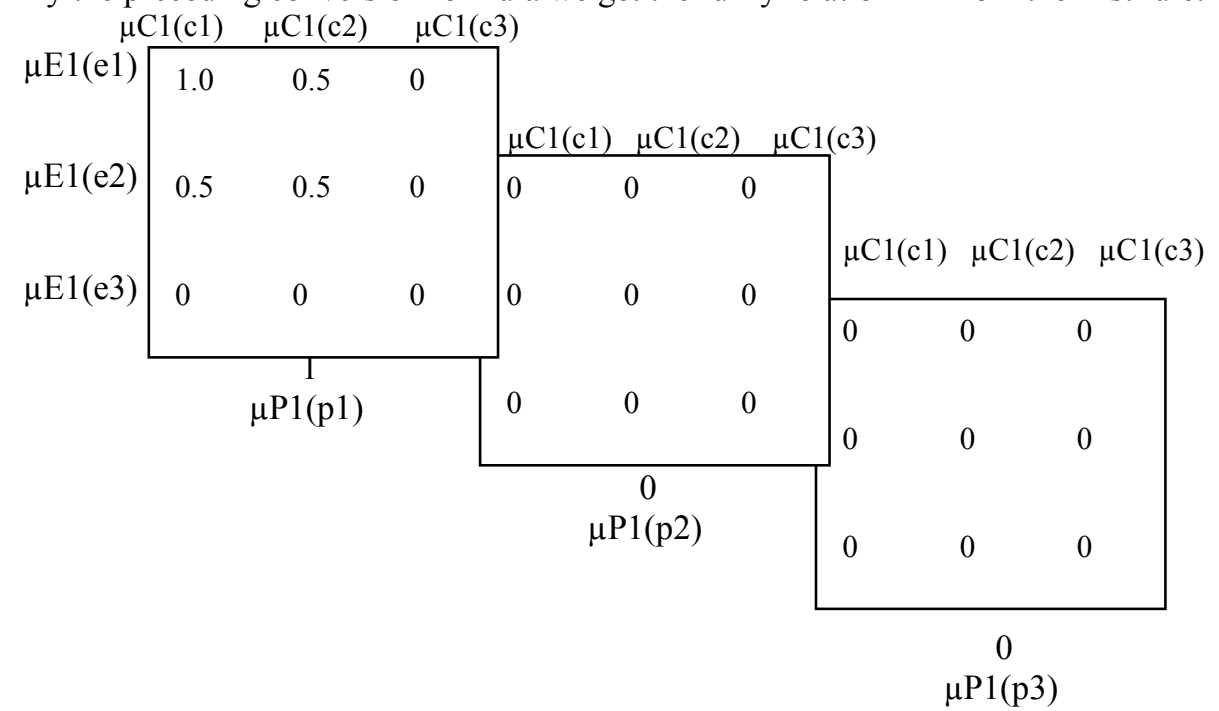

Similarly we can convert Rules 2, 3 and 4 into fuzzy relations R2, R3 and R4 accordingly. The total fuzzy relation $\mathrm{R}$ is given Mamdani's Method for compilation of fuzzy relations by

\section{$\mathrm{R}=\mathrm{R} 1 \mathrm{UR} 2 \mathrm{UR} 3 \mathrm{UR} 4$}

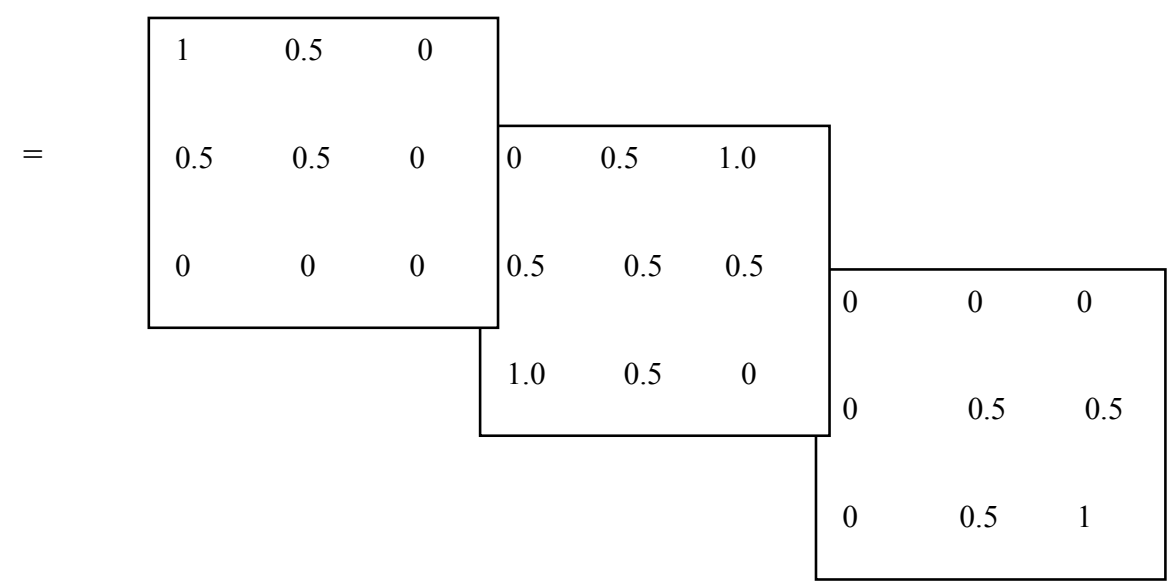

Let attitude of agent for buying the goods is high i.e. 1 and the competition in the market for that product is more i.e. 1. Such a situation can be described by fuzzy sets $E^{\prime}$ and $C^{\prime}$ as $E^{\prime}=[0,0,1] C^{\prime}=[0,0,1]$. Now the conclusion of the reasoning can be calculated by applying Mamdani's compositional rule of Inference[18] as follows $\mathrm{P}^{\prime}=\mathrm{C}^{\prime} \mathrm{o}(\mathrm{E}$ 'oR $)$. where $\mathrm{o}$ is the composition process. After implementing this we will get $\mathrm{P}^{\prime}=[0,0,1]$. Defuzzification of $\mathrm{P}^{\prime}$ by taking center of gravity with the weighted 
mean we get definite value for the bid displacement factor $\Delta \mathrm{P}$ as +100 . So the final bid price will be $\mathrm{P}=\mathrm{P}+\Delta \mathrm{P}$

\section{Conclusions}

In this paper we have designed a fuzzy competition and attitude based bidding strategy (FCA-Bid), which uses a soft computing method i.e. fuzzy logic technique to compute the final bid price based on the attitude of the agent and the competition in the market. Another unique idea presented in this paper is that to deal quantitatively the imprecision or uncertainty of multiple attributes of items to acquire in auctions, fuzzy set technique is used. The bidding strategy also allows for flexible heuristics both for the overall gain and for individual attribute evaluations. Specifically, the bidding strategy is adaptive to the environment as the agent can change the bid amount based its assessment of the various attributes of item, eagerness of agent as well as competition in the auction. The attitude of the agents is found with respect to the goods' attributes and the competition is calculated based on the number of bidders and the time elapsed for the auction. It was noticed that the strategies in which agent's behavior depends on attitudes and competition, are easily adaptable to the dynamic situations of the market $[14,17]$. An experimental evaluation is also conducted to find the final bid price on the bases of the historical auction records and by considering some set of rules for the attitude and the competition factors. In future we will investigate about the development of the bidding strategies for multiple auctions. We will also compare our bidding techniques with the other strategies to find out the relative strengths and the weaknesses.

\section{References}

1. Anthony, P. and N.R.Jennings.: Evolving bidding strategies for multiple auctions.: In Proceedings of 15th European Conference on Artificial Intelligence, pages 187--192, Netherlands (2002).

2. A. Byde, C. Priest, and N. R. Jennings. :Decision procedures for multiple auctions. In: Proceedings of the First International Joint Conference on Autonomous agents and Multiagent Systems, Bologana, Italy, pp.613--620 (2002).

3. Greenwald, A. and Stone, P.: Autonomous bidding agents in the trading agent competition. IEEE Internet Computing, pages 52--60(2001).

4. S.Murugesan. :Negotiation by Software agents in Electronic Marketplace, in:TENCON'00: Proceedings of the 2000 IEEE Region 10 Conference on Intelligent Systems and Technologies for the new Millenium, Kuala Lumpur Malasia, pp.286-$290,(2000)$. 
5. MA, H. Y. and LEUNG, H. F.: An Adaptive Attitude Bidding Strategy for Agents in Continuous Double Auctions. In: Electron. Commer. Res. Appl., 6(4). Elsevier, 383-398 (2007).

6. He, M., Leung, H., and Jennings, N.: A fuzzy logic based bidding strategy for autonomous agents in continuous double auctions. In:IEEE Transactions on Knowledge and data Engineering, 15(6):1345--1363 (2003).

7. Kowalcyzk, R. and Bui, V. :On fuzzy e-negotiation agents: Autonomous negotiation with incomplete and imprecise information. In: Proceedings Dexa e-Negotiation Workshop (2000).

8. Luo X., Jennings N., Shadbolt N., Leung H., and Lee J.: A fuzzy constraint based model for bilateral, multi-issue negotiation in semi-competitive environments. Artificial Intelligence, 148(1-2):53-102 (2003).

9. P.Anthony and N.R.Jennings. :Developing a bidding agent for multiple heterogeneous auctions. In:ACM transactions on Internet Technology, 3(3):185--217 (2003).

10. P.Stone, Littman, M., S.Singh, and M.Kearns. Attac-2000: An adaptive autonomous bidding agent.In: Journal of Artificial Intelligence Research, 15:189--206 (2001).

11. Kwang Mong Sim, Shi Yu Wang. In:IEEE Transactions on Systems, Man and Cybernetics- Part B: Cybernetics. Vol.34, No.3 (2004).

12. P.Faratin,C.Sierra and N.R.Jennings, :Negotiations decision functions for autonomous agents, In:Int. J. Robot. Auton Syst., Vol. 24, no.3,pp 159--182, (1998).

13. Madhu Lata Goyal, Jun Ma. Using Agents' Attitudes and Assessments in Automated Fuzzy Bidding Strategy. Proceeding of First International Conference on Agents and Artificial Intelligence, Porto, Portugal,(2009)

14. Huiye Ma, and Ho-Fung Leung .:An adaptive attitude bidding strategy for agents in continuous double auctions, Department of Computer Science and Engineering, The Chinese University of Hong Kong, Hong Kong, China, ( 2006).

15. Huiye Ma and Ho-fung Leung :Bidding Strategies in Agent-Based Continuous Double Auctions.In: Whitestein Series in Software Agent Technologies and Autonomic Computing pages 29--47

16. Srinivas K. Reddy and Mayukh Dass .:Modeling On-Line Art Auction Dynamics Using Functional Data Analysis. In:Statistical Science Vol. 21, No. 2, 179--193 Institute of Mathematical Statistics, (2006). 
17. Tagra H. Generic Architecture for Agents in E-Commerce (Auction).Ph. D dissertation. Indian Institute of Technology New Delhi, India,(2006).

18. Bapna, R., Jank, W. and Shmueli, G.: Price formation and its dynamics in online auctions. Working paper RHS-06-003, Smith School of Business, Univ. Maryland (2004).

19. Kazuo Tanaka: An Introduction to Fuzzy Logic for Practical Applications, Axel Springer Verlag,( 1991 ).

20. M. He and N. R. Jennings: Designing a successful trading agent using fuzzy techniques. In: IEEE Trans on Fuzzy Systems 12 (3) 389-410, (2003). 